\title{
Indecisión a las vacunas: una revisión sistemática para abordar el fenómeno en Latinoamérica
}

\author{
Vaccine hesitancy: A review to address the phenomenon in Latin America
}

\section{Hesitância vacina: uma revisão para enfrentar o fenômeno na América Latina}

\author{
Bruno Michel Bernal-Vaquera ${ }^{1 *}$ \\ (iD) https://orcid.org/0000-0002-0086-8534 \\ Alejandro Morales-Jinez ${ }^{2}$ \\ (D) https://orcid.org/0000-0002-2463-7482 \\ Norma Elvira Moreno-Pérez ${ }^{3}$ \\ (Dhttps://orcid.org/0000-0003-1829-3916
}

1. Estudiante de Maestría en Ciencias de Enfermería. Universidad de Guanajuato. Campus Celaya-Salvatierra. Celaya, Guanajuato, México,

2. Doctor en Ciencias de Enfermería. Universidad Autónoma de Coahuila. Escuela de Licenciatura en Enfermería Unidad Torreón. Torreón, Coahuila, México.

3. Doctora en Ciencias de Enfermería. Universidad de Guanajuato. Departamento de Enfermería Clínica. Celaya, Guanajuato, México.

*Autor para correspondencia: bruno.muchis@gmail.com

Recibido: 03/02/2020

Aceptado: 18/01/2021

\section{Resumen}

Introducción: En los últimos años se han desarrollado movimientos de personas, generalmente padres, que rechazan o están indecisas en cuanto al uso de las vacunas por la falta de conciencia de riesgos y la gravedad de las enfermedades prevenibles por vacunación, así como, por la difusión en las redes sociales de noticias falsas sobre la inocuidad y la eficacia de las vacunas. Investigaciones en países de Europa y América del Norte han descrito un término para estudiar este fenómeno el cual es Indecisión a las Vacunas, estas investigaciones son escasas en Latinoamérica. Objetivo: identificar instrumentos potencialmente válidos y confiables que puedan ser adaptados y modificados tanto al idioma español como al contexto de América Latina relacionados a la indecisión a las vacunas entre padres. Metodología: Se realizó una búsqueda en tres bases de datos PubMed, EBSCOhost y Biblioteca Virtual en Salud; con 
los términos descriptores de ciencias de la salud: "vaccine hesitancy", "parents" y "vaccine refutation". Resultados: Los resultados arrojaron 394 artículos de los cuales 19 fueron elegidos para el análisis. Los principales instrumentos identificados para medir la indecisión a las vacunas fueron los cuestionarios "Parental Attitudes About Childhood Vaccines" y "Vaccine Hesitancy Scale". Conclusiones: El cuestionario "Parental Attitudes About Childhood Vaccines" es el instrumento más usado y validado en diferentes idiomas para medir la indecisión a las vacunas.

Palabras clave: Vacunas; Padres; Negativa a la vacunación; Vacunación (DeCS).

\begin{abstract}
Introduction: In recent years there have been movements of people, usually parents, who reject or are undecided about the use of vaccines due to the lack of awareness of its risks and the severity of diseases that can be prevented by vaccination, as well as the dissemination of false news about the safety and efficacy of vaccines in the social networks. Research in countries in Europe and North America have come up with a term to study this phenomenon, this term is Vaccine Hesitancy. However, this research is scarce in Latin America. Objective: The objective of this review is to identify potentially valid and reliable instrument that can be adapted and modified both to Spanish and to the Latin America context, related to the indecision to use vaccines within parents. Methodology: A search was conducted on three PubMed, EBSCOhost and BVS databases; with the descriptive terms of health sciences: "vaccine hesitancy", "parents", and "vaccination refusal". The results yielded 394 articles of which 19 were chosen for the assessment.

Results: The main tools identified to measure vaccine hesitancy were the questionnaires named "Parental Attitudes about Childhood Vaccines" and "Vaccine Hesitancy Scale". Conclusion: "The Parent Attitudes on Childhood Vaccines" questionnaire is the most widely used and validated tool in different languages to measure vaccine hesitancy.
\end{abstract}

Key words: Vaccine; Vaccination refusal; Parents; Vaccination (DeCS).

\begin{abstract}
Abstrato
Introdução: Nos últimos anos, houve movimentos de pessoas, geralmente pais, que rejeitam ou estão indecisos quanto ao uso de vacinas devido à falta de consciência dos riscos e à gravidade das doenças evitáveis pelas vacinas, além da disseminação de redes sociais de notícias sobre a segurança e eficácia das vacinas. Pesquisas em países da Europa e América do Norte descreveram um termo para estudar esse fenômeno, que é a vacinação vacinal, essas investigações são escassas na América Latina. Objetivo: O objetivo desta revisão é pesquisar na literatura científica estudos onde você estudou a hesitação da vacina entre os pais e identificar ferramentas potencialmente válidas e confiáveis que possam ser adaptadas e modificadas tanto no idioma espanhol quanto no contexto da América Latina. Metodologia: Uma pesquisa foi realizada em três bancos de dados PubMed, EBSCO Host e BVS; com os termos descritivos das ciências da saúde: "vaccine hesitancy", "parents" e "vaccination refusal". Resultados: Os resultados renderam 394 artigos, dos quais 19 foram escolhidos para a análise. A principal ferramenta identificada para medir a hesitação vacinal foram os questionários " Parental Attitudes About Childhood Vaccines" e "Vaccine Hesitancy Scale". Conclusões: O questionário "The Parent Attitudes on Childhood Vaccines" é a ferramenta mais amplamente utilizada e validada em diferentes idiomas para medir a hesitação da vacina.
\end{abstract}

Palavras-chave: Vacina; Recusa de vacinação; Pais; Vacinação (DeCS). 


\section{Introducción}

En las últimas décadas, la vacunación a nivel mundial refleja un progreso limitado, en el informe de la Organización Mundial de la Salud (OMS) la cobertura mundial se mantiene aproximadamente en el 86\% y se estimó que más de 19 millones de lactantes no fueron vacunados contra el tétanos, la difteria y tos ferina ${ }^{(1)}$. De acuerdo con el informe 2019 de Inmunización en las Américas ${ }^{(2)}$, en la región, hay brotes de sarampión y difteria debido a la baja cobertura de vacunas contra el sarampión, rubeola y parotiditis (SRP) $82 \%$ y difteria, tétanos y tos ferina (DPT) $88 \%$, otras vacunas también presentan baja cobertura como la hepatitis B en el recién nacido con $80 \%$, rotavirus $79 \%$ y vacuna antipoliomielítica oral en $87 \%$. Aunado a lo anterior, la comunicación con la sociedad también se ha vuelto complicada por la falta de conciencia de riesgos y la gravedad de las Enfermedades Prevenibles por Vacunación (EPV) así como, por la difusión en las redes sociales de noticias falsas sobre la inocuidad y la eficacia de las vacunas ${ }^{(3-9)}$.

Debido a estos acontecimientos existe el riesgo de aumento de brotes de EPV, por lo cual, el personal del área de la salud especialmente enfermería, al ser responsable de la ejecución de programas de vacunación en varios países, debe diseñar estudios para generar conocimiento y difundir que las vacunas son como cualquier medicamento probado antes de su comercialización y uso en la población que es susceptible a causar mínimos efectos secundarios o Eventos Atribuibles a la Vacunación o Inmunización (ESAVI), no obstante, el beneficio individual y colectivo es muy superior a los supuestos riesgos ${ }^{(9)}$.

De acuerdo con algunos estudios ${ }^{(3-9)}$, la negativa de los padres a que sus hijos sean vacunados se centra en factores valóricos, falta de información, una profunda desconfianza frente a las instituciones de salud, desconocimiento de los padres en cuanto a las vacunas, así como sus beneficios, lo cual dificulta la toma de decisiones sobre la vacunación de los niños y, en consecuencia, se cree que la vacunación es obligatoria por parte de las autoridades, motivado por intereses particulares.

McDonald y su Grupo de Expertos en Asesoramiento Estratégico (SAGE) ${ }^{(10)}$ han realizado y revisado estudios de investigación para generar un término y dar significado a un constructo bajo el que se pueda estudiar esta situación, el término más asociado en idioma inglés es "Vaccine Hesitancy" (VH). Siguiendo el diccionario inglés-español (11) 
"Vaccine" se traduce como "vacuna" y "hesitancy" como "vacilación o indecisión", en conjunto la traducción al español se puede tomar como "indecisión a las vacunas (IV)". La definición por el SAGE Working Group en VH es "la demora en la aceptación o rechazo de la vacunación a pesar de la disponibilidad de servicios de vacunación" (10).

Países de Europa, Asia y Norte América han iniciado a realizar investigaciones para abordar esta situación, dando resultados positivos en la identificación de este grupo de personas ya que conforman un grupo mucho más grande que aquellos que rechazan totalmente las vacunas ${ }^{(10)}$, descubriendo que son más susceptibles al cambio de comportamiento porque tienden a buscar información con los responsables de la vacunación de sus hijos. Sin embargo, en América Latina estos estudios son escasos pero necesarios, ya que los padres que presentan VH son un grupo de interés porque representan una oportunidad para trabajar en la forma de detectar y abordar las dudas e inseguridades y ayudar a idear intervenciones eficaces para incrementar la cobertura de la vacunación.

Por lo tanto, el objetivo de esta revisión fue buscar en la literatura científica estudios donde se haya abordado el fenómeno VH e identificar herramientas válidas y confiables para reconocer y medir VH, que puedan ser adaptadas al idioma español y al contexto de América Latina.

\section{Metodología}

Se llevó a cabo una revisión sistemática iniciando con el planteamiento de la interrogante que guió la revisión, donde el problema es $\mathrm{VH}$, la intervención es la medición del fenómeno con instrumentos válidos y confiables, y se llevó a cabo la comparación entre ellos de acuerdo con su confiabilidad y resultados al ser traducidos a otro idioma y realizar la medición en la población. La pregunta se planteó de la siguiente manera: ¿Existe evidencia científica de herramientas válidas y confiables para medir la indecisión a las vacunas que puedan ser susceptibles de adaptación al idioma español? Se realizó la búsqueda para identificar estudios que investigaron la VH en padres considerando los términos "vaccine hesitancy" y "parents" con el operador boleano "AND". Los resultados incluían numerosos estudios que analizaban el rechazo a la vacunación, por lo que se agregó a la búsqueda "vaccine refutation" con el operador boleano "NOT". La búsqueda se limitó a artículos de texto completo publicados en los últimos cinco años en las bases de datos 
PubMed, Biblioteca Virtual en Salud (BVS) y EBSCO Host con el protocolo de búsqueda: "vaccine hesitancy AND parents NOT vaccine refutación" (Tabla 1).

Tabla 1. Protocolo de Búsqueda en base de datos

\begin{tabular}{cc}
\hline $\begin{array}{c}\text { Base de } \\
\text { Datos }\end{array}$ & Protocolo de Búsqueda \\
\hline & $($ (("vaccines"[MeSH Terms] OR "vaccines"[All Fields] OR "vaccine"[All Fields]) AND hesitancy[All Fields]) \\
AND ("parents"[MeSH Terms] OR "parents"[All Fields])) NOT ("vaccination refusal"[MeSH Terms] OR \\
("vaccination"[All Fields] AND "refusal"[All Fields]) OR "vaccination refusal"[All Fields]) AND \\
("2014/10/11"[PDat] : "2019/10/09"[PDat]) \\
EBSCOhost \\
vaccine hesitancy AND parents NOT vaccination refusal. \\
Fecha de publicación: 20141001-20191031 \\
Modos de búsqueda - Booleano/Frase \\
(tw:(vaccine hesitancy)) AND (tw:(parents)) AND NOT (tw:(vaccination refusal)) \\
\hline
\end{tabular}

Fuente: Elaboración propia

La selección de artículos consistió en que una de las variables en los estudios fuera VH medida en padres, siguiendo la revisión de la metodología de los artículos, se encontró que algunos hacían la medición de la variable a través de un ítem de autoinforme, el cual preguntaba a los padres si se consideraban indecisos respecto a las vacunas. Esto dio una nueva pauta para eliminar aquellos estudios en los que la VH fuera medida a través del autoinforme de los padres (las limitaciones de estos estudios consideraron que había riesgo de sesgo de deseabilidad social y los padres podían no haber contestado honestamente) y se seleccionaron aquellos que la midieran través de un instrumento desarrollado. Así mismo, se revisaron las variables que pueden ser medidas y que cobran relevancia junto a la VH ya sean variables sociodemográficas u otras variables de estudio propiamente.

Los artículos fueron analizados y la información necesaria fue concentrada en una matriz de datos, donde además de los datos de identificación del artículo y autores, se identificaron los métodos del estudio, la definición que se establece de $\mathrm{VH}$, dimensiones, identificación del instrumento de medición, resultados, limitaciones y conclusión. 


\section{Resultados}

El resultado de la búsqueda en las tres bases de datos arrojó un total de 394 artículos, se seleccionaron 41 y se eliminaron por duplicación 18, dejando 23 artículos, todos ellos publicados en idioma inglés. Se eliminaron 4 artículos debido a que la variable VH era medida por un ítem de autoinforme. El número total de artículos para el análisis fue de $n=19$ (Figura 1).

Figura 1. Resultados de búsqueda

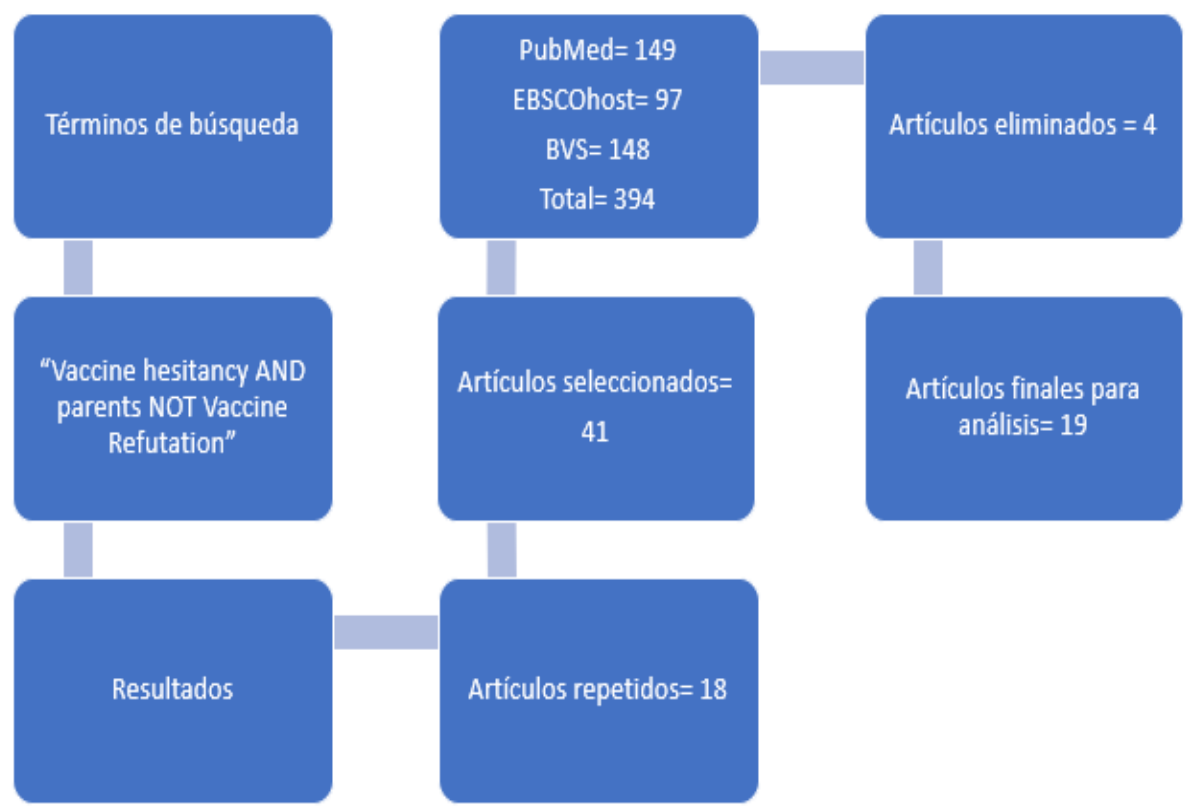

Fuente: Elaboración propia

Del total de artículos seleccionados para el análisis, el 100\% se publicaron en idioma inglés, 9 estudios se realizaron en Estados Unidos, 2 en Canadá, 3 en Europa, 3 en Asia, 1 en América Latina y 1 se realizó en varios países de diferentes regiones. 9 de los estudios se enfocaron en desarrollar un cuestionario o modificar uno existente para medir VH en padres, y 10 fueron investigaciones de tipo descriptivos, correlacional o ensayos clínicos con intervención con o sin aleatorización de la muestra, teniendo a este constructo entre sus variables de estudio (Tabla 2). 
Tabla 2. Artículos analizados




Tabla 2 continuación...)

\begin{tabular}{|c|c|c|c|c|c|}
\hline $\begin{array}{l}\text { Reliability and validity of a } \\
\text { survey to identify vaccine } \\
\text { hesitancy among parents in } \\
\text { Changxing county, Zhejiang } \\
\text { province (17) }\end{array}$ & $\begin{array}{l}\text { Yu Hu, Yaping Chen, } \\
\text { Hui Liang, Ying Wang }\end{array}$ & $\begin{array}{l}\text { Evaluar la validez y } \\
\text { confiabilidad de una } \\
\text { encuesta para } \\
\text { identificar IV entre } \\
\text { los padres }\end{array}$ & $\begin{array}{l}\text { Survey of vaccine } \\
\text { hesitancy }\end{array}$ & 24,9 & III \\
\hline $\begin{array}{l}\text { Physician Communication } \\
\text { Training and Parental Vaccine } \\
\text { Hesitancy: A Randomized Trial } \\
\text { (19) }\end{array}$ & Nora B. Henrikson, et al. & $\begin{array}{l}\text { Reducir la IV en } \\
\text { madres de niños } \\
\text { atendidos por } \\
\text { medicos } \\
\text { capacitados e } \\
\text { incrementar la } \\
\text { confianza del } \\
\text { médico en } \\
\text { comunicación sobre } \\
\text { vacunas }\end{array}$ & PACV & $12.6 / 9.8^{h}$ & I \\
\hline $\begin{array}{l}\text { Longitudinal Trends in Vaccine } \\
\text { Hesitancy in a Cohort of } \\
\text { Mothers Surveyed in } \\
\text { Washington State, 2013-2015 } \\
\text { (20) }\end{array}$ & $\begin{array}{l}\text { Nora B. Henrikson, } \\
\text { Melissa L. Anderson, } \\
\text { Douglas J. Opel, John } \\
\text { Dunn, Edgar K. } \\
\text { Marcuse, David C. } \\
\text { Grossman }\end{array}$ & $\begin{array}{l}\text { Evaluar la tendencia } \\
\text { en la IV de los } \\
\text { padres durante los } \\
\text { primeros } 2 \text { años de } \\
\text { vida de sus hijos en } \\
\text { una cohorte de } \\
\text { madres en el estado } \\
\text { de Washington }\end{array}$ & PACV & $9.7^{d}$ & II-3 \\
\hline $\begin{array}{l}\text { Previsit Screening for Parental } \\
\text { Vaccine Hesitancy: A Cluster } \\
\text { Randomized Trial (21) }\end{array}$ & Douglas J. Opel, et al. & $\begin{array}{l}\text { Evaluar el efecto de } \\
\text { identificar padres } \\
\text { con IV, antes de sus } \\
\text { visitas de } \\
\text { supervisión de salud } \\
\text { en la aplicación de } \\
\text { vacunas de la } \\
\text { infancia }\end{array}$ & PACV & $19.6^{d}$ & $\mathrm{II}-3$ \\
\hline $\begin{array}{l}\text { Validation study of the Parent } \\
\text { Attitudes About Childhood } \\
\text { Vaccines (PACV) } \\
\text { questionnaire: The Malay } \\
\text { version (22) }\end{array}$ & $\begin{array}{l}\text { Haizlene Abd Halim, } \\
\text { Suraya Abdul-Razak, } \\
\text { Mazapuspavina Md } \\
\text { Yasin, Mohamad Rodi } \\
\text { Isa }\end{array}$ & $\begin{array}{l}\text { Adaptar y traducir } \\
\text { el cuestionario } \\
\text { original PACV del } \\
\text { idioma inglés al } \\
\text { idioma malayo y } \\
\text { examinar sus } \\
\text { propiedades } \\
\text { psicométricas }\end{array}$ & $\begin{array}{l}\text { PACV versión } \\
\text { malaya }\end{array}$ & NA & III \\
\hline $\begin{array}{l}\text { Comparative analysis of the } \\
\text { Parent Attitudes about } \\
\text { Childhood Vaccines (PACV) } \\
\text { short scale and the five } \\
\text { categories of vaccine } \\
\text { acceptance identified by Gust } \\
\text { et al (23) }\end{array}$ & $\begin{array}{l}\text { Omolade Oladejo, } \\
\text { Kristen Allen, Avnika } \\
\text { Amin, Paula M. Frew, } \\
\text { Robert A. Bednarczyk, } \\
\text { Saad B. Omer }\end{array}$ & $\begin{array}{l}\text { Evaluar cómo las } \\
\text { categorías de } \\
\text { aceptación de } \\
\text { vacunas de Gust et } \\
\text { al. corresponden a } \\
\text { la escala corta de } \\
\text { PACV }\end{array}$ & $\begin{array}{l}\text { PACV escala corta/ } \\
\text { HealtStyles survey } \\
\text { adoptadas del } \\
\text { análisis de Gust et } \\
\text { al. }\end{array}$ & $13 / 15.2^{c}$ & III \\
\hline
\end{tabular}


(Tabla 2 continuación...)

\begin{tabular}{|c|c|c|c|c|c|}
\hline $\begin{array}{l}\text { Measuring vaccine hesitancy: } \\
\text { Field testing the WHO SAGE } \\
\text { Working Group on Vaccine } \\
\text { Hesitancy survey tool in } \\
\text { Guatemala }\end{array}$ & $\begin{array}{l}\text { Gretchen J. Domek, et } \\
\text { al. }\end{array}$ & $\begin{array}{l}\text { Proporcionar } \\
\text { información sobre } \\
\text { la comprensión } \\
\text { compartida del } \\
\text { constructo VHS } \\
\text { usando la } \\
\text { herramienta en } \\
\text { diversos entornos } \\
\text { globales }\end{array}$ & VHS & 1,1 & III \\
\hline $\begin{array}{l}\text { Vaccine hesitancy among } \\
\text { parents in a multi-ethnic } \\
\text { country, Malaysia }\end{array}$ & $\begin{array}{l}\text { Fatin Shaheera Mohd } \\
\text { Azizi, Yueting Kew, } \\
\text { Foong Ming Moy }\end{array}$ & $\begin{array}{l}\text { Evaluar la } \\
\text { confiabilidad del } \\
\text { cuestionario PACV } \\
\text { en idioma malayo; } \\
\text { para determinar la } \\
\text { prevalencia de IV } \\
\text { entre los padres y } \\
\text { su asociación con } \\
\text { las características } \\
\text { sociodemográficas } \\
\text { de los padres }\end{array}$ & $\begin{array}{l}\text { PACV versión } \\
\text { malaya }\end{array}$ & 11,6 & III \\
\hline $\begin{array}{l}\text { Implementing vaccine } \\
\text { hesitancy screening for } \\
\text { targeted education (26) }\end{array}$ & $\begin{array}{l}\text { John T. Connors, Eric A. } \\
\text { Hodges, Jennifer } \\
\text { D'Auria, } \\
\text { Laura Windham }\end{array}$ & $\begin{array}{l}\text { Determinar si } \\
\text { usando una } \\
\text { herramienta de } \\
\text { detección (VHS) en } \\
\text { conjunto con } \\
\text { proveer pláticas } \\
\text { abordando las } \\
\text { preocupaciones de } \\
\text { los padres afecta la } \\
\text { intención de los } \\
\text { padres a vacunar }\end{array}$ & VHS & $18.9^{d}$ & II-3 \\
\hline
\end{tabular}


(Tabla 2 continuación...)

\begin{tabular}{|c|c|c|c|c|c|}
\hline $\begin{array}{l}\text { Vaccine hesitancy in the } \\
\text { French population in 2016, } \\
\text { and its association with } \\
\text { vaccine uptake and perceived } \\
\text { vaccine risk-benefit balance } \\
\text { (28) }\end{array}$ & Dominique Rey, et al. & $\begin{array}{l}\text { Estimar la } \\
\text { prevalencia y } \\
\text { correlaciones } \\
\text { sociodemográficas } \\
\text { de IV en subgrupos } \\
\text { de población } \\
\text { francesa, e } \\
\text { investigar la } \\
\text { asociación de IV } \\
\text { con la aplicación de } \\
\text { vacunas y la } \\
\text { percepción de la } \\
\text { balanza riesgo } \\
\text { beneficio para } \\
\text { cuatro vacunas }\end{array}$ & 3 ítems de VHS & $46 / 48^{i}$ & III \\
\hline $\begin{array}{l}\text { Prevalence of Vaccine } \\
\text { Hesitancy Among Expectant } \\
\text { Mothers in Houston, Texas }{ }^{(30)}\end{array}$ & $\begin{array}{l}\text { Rachel M. Cunningham, } \\
\text { Charles G. Minard, } \\
\text { Danielle Guffey, Laurie } \\
\text { S. Swaim, Douglas J. } \\
\text { Opel, Julie A. Boom }\end{array}$ & $\begin{array}{l}\text { Evaluar la } \\
\text { prevalencia de IV } \\
\text { entre futuros padres } \\
\text { que recibieron } \\
\text { atención obstétrica } \\
\text { en clínicas de } \\
\text { Houston, Texas. }\end{array}$ & $\begin{array}{l}\text { PACV Modificada } \\
\text { para futuros } \\
\text { padres. }\end{array}$ & 8 & III \\
\hline
\end{tabular}

\footnotetext{
a Indecisión a la vacunación

${ }^{b}$ Parental Attitudes about Childhood Vaccines

${ }^{c} \mathrm{NI}$ nivel intermedio/NAL nivel alto de IV

${ }^{\mathrm{d}}$ En estudios con pre y postest se tomó el resultado del pretest.

e No Aplica

${ }^{f}$ Niveles de evidencia por tipo de diseño (USPSTF)

9 Vaccine Hesitancy Scale

h Grupo Control/Grupo Intervención

i Padres de niños/padres de mujeres adolescentes
}

Fuente: Elaboración propia

La definición de VH es constante en los estudios siguiendo en su mayoría la definición del SAGE Working Group agregando que es un fenómeno complejo y específico de un contexto que cambia con el tiempo, lugar y el tipo de vacuna. Los cuestionarios o escalas utilizadas que sufrieron modificación para su uso fueron: Parental Attitudes About Childhood Vaccines (PACV) $n=12^{(12,15,16,18-22,25,27,29,30)}$ Vaccine Hesitancy Scale (VHS) $n=5^{(13,14,24,26,28)}$ Survey of 
Vaccine Hesitancy $n=1^{(17)}$ y un estudio comparativo entre el PACV y Healt Styles Survey adoptadas del análisis de Gust et al. ${ }^{(23)}, n=1$.

Al consultar la confiabilidad de los instrumentos a través del Alfa de Cronbach, se encontró que el PACV en la versión original en inglés, consta de quince ítems en tres factores: "seguridad y eficacia", "actitudes generales" y "conductas" con alfa de 0,74 0,84, y 0,74 respectivamente, que miden dimensiones como conductas de vacunación, creencias acerca de la seguridad y eficacia de las vacunas, actitudes acerca de la obligatoriedad y exenciones de vacunación, y confianza. La versión malaya del PACV con un Alfa de Cronbach general de 0,77 y la versión italiana con $0,91^{(12,15,18-22,25,27,29,30)}$

El PACV también fue traducido al español, fue revisado por expertos y probado en una población de habla hispana en Estados Unidos ${ }^{(16)}$. Sin embargo, no se evaluó la validez y confiabilidad del instrumento, pero el hecho de que haya sido revisado por expertos puede otorgar cierto grado de validez. De igual manera el PACV se modificó para ser aplicado en padres de adolescentes con un factor llamado "seguridad y dudas" (18). Para la evaluación del puntaje del PACV fue de 0 a 100 puntos, interpretados de dos maneras; la primera: 0-29 bajo nivel de IV, 30-49 puntos nivel intermedio y $>50$ nivel alto, la segunda manera: $<50$ puntos sin presencia de IV y $>50$ con VH.

La escala VHS desarrollada por el SAGE Working Group en VH es compendio de diferentes versiones las cuales son VHS versión 1,0, VHS escala de Likert de 5 puntos VHS encuesta de preguntas abiertas ${ }^{(14)}$, a pesar de que las escalas no han sido validadas, en algunos estudios en los que se utilizó se tuvieron resultados favorables en la identificación de padres con VH, se hacen recomendaciones para trabajar en ello. La escala evalúa dimensiones como complacencia, conveniencia y confianza ${ }^{(13,14,24,26,28)}$. Dando un rango de puntaje en la escala de tipo likert de 0 a 50. Una versión modificada en Reino Unido del VHS de 9 ítems consta de factores como "falta de confianza y percepción de riesgo" ${ }^{\prime 13)}$.

El instrumento desarrollado en China, Survey of Vaccine Hesitancy tiene un total de quince ítems, agrupados en tres factores: "conductas" con alfa de Cronbach 0,71, "seguridad y eficacia" con 0,83, y "actitudes" con 0,72 (17). 
En los estudios en los que su objetivo no sólo era desarrollar un instrumento de medición, sino detectar a los padres con HV a través de los instrumentos ya desarrollados, así como aplicar intervenciones para solventar el problema, tuvieron buenos resultados identificando a esta población, ya que en los resultados de los estudios se muestra que desde $1 \%$ hasta $48 \%$ de la población pudo ser identificada con VH (Tabla 2).

Las variables sociodemográficas de interés en los estudios fueron el género, edad, educación, ingresos económicos, número de hijos, edad de los hijos, empleo y estado civil. Otras variables de estudio fueron el estado de vacunación del hijo y si habían retrasado o rechazado alguna vez la administración de al menos una vacuna, la percepción de las EPV y ESAVI se emplearon de forma correlacional y predictiva, es decir si el resultado obtenido de los instrumentos puede tener efecto en el estado de vacunación del hijo y la probabilidad de que retrasen o rechacen en algún momento la administración de alguna vacuna $(15,17,18,23,25,27-30)$.

Las limitaciones de los estudios en general fueron en torno a la falta de validación del instrumento, el tamaño de la muestra con bajo porcentaje de participación a lo esperado o no contaban con muestras representativas de la región del estudio limitando la generalización de los resultados, la posible presencia de sesgo de deseabilidad social en variables como el estatus de vacunación del niño y retraso o rechazo de la aplicación de alguna vacuna ${ }^{(12,14,16,18-}$ 22,24-30)

\section{Discusión}

El PACV fue el instrumento más utilizado entre los estudios analizados y cuenta con validez y confiabilidad probada, ha sido modificado para usarse en situaciones específicas como el tipo de vacuna, padres en diversas situaciones y en distintos países del mundo. En contexto específico de una vacuna, se realizó un estudio para determinar la indecisión hacia la vacuna anti-influenza por parte de los padres durante la hospitalización del hijo (27), haciendo modificaciones mínimas en el PACV sólo para resaltar y dejar en claro la orientación hacia esta vacuna. Los resultados muestran la presencia de $\mathrm{VH}$ ya que el $53 \%$ de los padres declinaron la vacuna de la influenza durante la hospitalización, en el 24\% de los encuestados se obtuvo un puntaje alto de indecisión $>50$, y se encontró que una proporción mayor de los padres con puntajes altos declinaron la vacunación en comparación con los que tenían puntajes bajos. Estos resultados 
de VH en vacuna contra la influenza tienen concordancia con la definición ya que menciona que puede ser específica de cada vacuna.

El PACV también fue aplicado en futuras madres ${ }^{(30)}$ y detectó que 1 de cada 12 (8\%) mujeres embarazadas presenta $\mathrm{VH}$, en un estudio de cohorte ${ }^{(20)}$ se encontró una disminución significativa entre y la proporción de madres con VH 9,7\% al nacimiento del hijo vs 5,9\% a los 24 meses de edad. Los resultados sugieren que la VH puede disminuir con el tiempo para muchos padres a medida que se acumula experiencia materna con la vacunación. En un estudio usando una versión modificada del PACV para padres de adolescentes ${ }^{(18)}$ se encontró que sólo 39\% expresaron preocupación por las enfermedades prevenibles y $41 \%$ expresó preocupación por serios efectos secundarios, y $46 \%$ estaban en desacuerdo con que los adolescentes pueden recibir todas las vacunas recomendadas en una sola visita, sólo dos ítems del instrumento fueron asociados con la baja vacunación entre adolescentes. Estos resultados muestran que los niveles de VH están presentes en diferente tiempo, contexto como en futuras madres, padres de niños o adolescentes y de nuevo concuerda con lo establecido en la definición de VH.

El PACV traducido a diferentes idiomas y utilizado en diferentes países, muestra resultados donde la indecisión a las vacunas está presente, en Canadá se encontró entre madres la presencia de VH ya que la media del puntaje fue de 27 , con $28,6 \%$ con un puntaje intermedio de indecisión y un $15 \%$ con un puntaje alto de vacilación ${ }^{(15)}$. También se encontró una tendencia lineal significativa entre el puntaje de VH y la intención de vacunar a su hijo. En un estudio con intervención basada en entrevistas motivacionales para reducir la $\mathrm{VH}{ }^{(12)}$ se describió que después de la intervención, la población con VH más baja aumentó de 55,9\% a 78,8\% (aumento del 41\%), mientras que aquellos con niveles intermedios disminuyeron de $44,1 \%$ a $21,1 \%$, el 15,6\% de la población mostró un nivel alto y disminuyó a sólo 5,2\% después de la intervención. Se puede observar una proporción similar en los dos estudios en la proporción de padres que presentan niveles altos de indecisión tomando en cuenta la medición pretest en el estudio intervención. En Italia (29) la media para el puntaje del PACV en italiano fue de 45,8 con $34,7 \%$ padres con niveles altos de $\mathrm{VH}$, a los que fueron asociados variables como ser madre, joven, bajo nivel de educación, creer que las EPV no son graves, preocupación por efectos secundarios. Y describieron que la razón más frecuente para rechazar o retrasar las vacunas 
fue la falta de recomendación por parte de los pediatras (35,1\%). En Malasia de acuerdo con el PACV ${ }^{(25)}$ el 11,6\% fueron catalogados como padres con VH y fueron asociados con las siguientes características: padres sin empleo, con hijos menores, no musulmanes y madres esperando su primer hijo, lo que sugiere que los factores asociados a la $\mathrm{VH}$ pueden variar respecto a las características de la población de cada país. En el estudio efectuado en Washington donde evaluaron una intervención de entrenamiento en comunicación para médicos ${ }^{(19)}$, se detectó al inicio VH en los grupos control (12,6\%) e intervención (9,8\%). El funcionamiento del PACV en diferentes países e idiomas y su capacidad para detectar a padres con VH es óptimo y demuestra ser un instrumento válido y confiable en diferentes lugares, contextos más allá de la puntuación del alfa de Cronbach, así mismo, el lugar diferenciará las variables sociodemográficas y de estudio que puedan ser asociadas y relacionadas con los puntajes del PACV.

En cuanto al VHS es un instrumento que se encuentra en desarrollo, el cual tiene por objetivo ser un instrumento global avalado por la OMS para medir la VH ${ }^{(10,14)}$, sin embargo, en el Reino Unido ${ }^{(13)}$ en un estudio de modificación de la encuesta se encontró que el 4\% de la muestra respondió con indecisión en todos los ítems y el 19\% al menos en uno. En Guatemala ${ }^{(24)}$, la escala VHS en tipo Likert mostró que ningún padre había rechazado alguna vacuna, y sólo $1,1 \%$ han vacilado alguna vez, ninguno piensa en alguna razón para no vacunar, pero sí se detectaron pensamientos de indecisión, de ellos $3(0,4 \%)$ no creen que las vacunas protejan de enfermedades graves, el $59 \%$ de los padres piensa que los hijos de otras personas no tienen todas las vacunas recomendadas, lo cual sugiere que la encuesta tiene limitaciones para identificar comportamientos de indecisión y puede ser difícil de comprender pero muestra que pensamientos y creencias hacia la $\mathrm{VH}$ pueden estar presentes. Sin embargo, en Francia ${ }^{(28)}$ se usaron 3 ítems de la encuesta y se identificó presencia de VH entre padres de niños y mujeres adolescentes, respecto a vacunación de sus hijos fue de $46 \%$ al $48 \%$ y fue significativamente asociada a variables demográficas como educación, bajo ingreso, y una pobre percepción en EPV. En padres con al menos un hijo de 10 a 15 años fueron más propensos a ser indecisos que padres con hijos menores, los niveles fueron significativamente más bajos en personas con reporte de vacunación de sarampión y hepatitis en sus hijos. Esto sugiere que la VHS tiene los elementos y capacidades para poder detectar la $\mathrm{VH}$, aunque son necesarios más estudios sobre todo de validación, para que pueda cumplir 
completamente con el objetivo de ser utilizado globalmente como lo recomienda el SAGE Working Group en VH de la OMS.

Las investigaciones donde se desarrolló alguna intervención enfocada a modificar los niveles de VH, a través de técnicas basadas en entrevistas motivacionales, entrenamiento en comunicación para médicos para tratar con las inseguridades de los padres hacia la vacunación, detección precoz de los padres con indecisión antes de la visita de salud de su hijo y proveer pláticas acerca de las preocupaciones de los padres ${ }^{(12,19,21,26)}$. El éxito o fracaso del estudio se debió al diseño de la intervención y no a la capacidad de detectar los niveles de VH. Las limitaciones en cuanto a los instrumentos fue que en algunos estudios donde se modificó el instrumento no se evaluó la validez y confiabilidad, pudiendo afectar la validez del constructo. Los autores mencionaron que a pesar de no haber medido la validez y confiabilidad en algunas versiones modificadas de los instrumentos y considerando los resultados, son herramientas que permitieron detectar a padres que presentan $\mathrm{VH}(14,16,22,24,26,29,30)$.

\section{Conclusiones}

El PACV de Opel es el instrumento confiable y validado, siendo usado y modificado en diferentes contextos midiendo de forma efectiva la VH. El VHS del SAGE Working Group en VH es un instrumento potencial para medir la VH, se recomienda hacer estudios de validación de esta escala para ampliar las opciones para evaluar este fenómeno. Las variables que principalmente se asociaron a la VH son el estado de vacunación del hijo, educación, número de hijos, baja percepción de EPV. Los estudios sobre $\mathrm{VH}$ en América Latina son escasos, pero necesarios, ya que pueden contribuir a elaborar estrategias para mejorar la cobertura de vacunación en la región de Las Américas.

\section{Conflicto de intereses}

Los autores declaran no tener algún conflicto de intereses en la realización y publicación de este trabajo.

\section{Financiamiento}

No se recibió ningún financiamiento para la elaboración del presente trabajo. 


\section{Referencias bibliográficas}

1. OMS: Organización Mundial de la Salud [Internet] Suiza. 2019. [Citado 2019 Nov 06]. Organización Mundial de la Salud Cobertura Vacunal. [aprox. 1 pantalla]. Disponible en: https://www.who.int/es/news-room/factsheets/detail/immunization-coverage

2. Organización Panamericana de la Salud. Inmunización en las Américas, Resumen 2019. [Internet]. Washington: Inmunización Integral de la Familia, Familia, Promoción de la Salud y Curso de Vida; 2019 [Citado 2019 Nov 06]. Disponible en: https://www.paho.org/hq/index.php?option=com_docman\&view=download\&category_slug=folletoinmunizaciones-2646\&alias=50554-inmunizacion-en-las-americas-resumen-2019\&ltemid=270\&lang=es

3. Lopera Pareja EH. El movimiento antivacunas. Argumentos, causas y consecuencias. [Internet] Madrid: Catarata, 2016 [Citado 2019 Nov 06]. Disponible en:

https://www.oei.es/historico/divulgacioncientifica/IMG/pdf/antivacunas_tripas.pdf

4. Cuesta Cambra U, Gaspar Herrero S. La "reputación online" de la información de vacunas en internet. Historia y Comunicación Social. 2014 [Citado 2019 Nov 06];19:15-29. Disponible en:

http://dx.doi.org/10.5209/rev_HICS.2014.v19.45007

5. Martínez Diz S, Martínez Romero M, Fernández Prada M., Cruz Piqueras M., Molina Ruano R, Fernández Sierra MA. Demandas y expectativas de padres y madres que rechazan la vacunación y perspectiva de los profesionales 43 sanitarios sobre la negativa a vacunar. An Pediatr. [Internet] 2014 [Citado 2019 Nov 06];80(6):370-378. Disponible en: http://www.sciencedirect.com/science/article/pii/S1695403313003779

6. Véliz L, Campos C, Vega P. Conocimiento y actitudes de los padres en relación a la vacunación de sus hijos. Rev. chil. infectol. [Internet]. 2016 [Citado 2019 Nov 06];33(1):30-37. Disponible en:

https://scielo.conicyt.cl/scielo.php?script=sci_arttext\&pid=S0716-

7. Cruz Piqueras M, García de Cortazar AR, Hortal Carmona J, Padilla Bernaldez. Reticencia vacunal: análisis del discurso de madres y padres con 44 rechazo total o parcial a las vacunas. Gac. Sanit. [Internet] 2019 [Citado 2019 Nov 06];33(1):53-59. Disponible en:

https://www.sciencedirect.com/science/article/pii/S0213911117301838?via\%20\%3Dihub

8. Guadarrama Orozco JH, Vargas López G, Viesca Treviño C. Decisiones de los padres que no arriesgan la vida de sus hijos, pero que los exponen a daños serios: no a las vacunas. Bol. Med. Hosp. Infant. Mex. [Internet] 2015 [Citado 2019 Nov 06];72(5):353-357. Disponible en: http://dx.doi.org/10.1016/j.bmhimx.2015.09.007

9. Domínguez A. Astray J, Castilla J,Godoy P, Tuells J, Barrabeig I. Falsas creencias sobre vacunas. Aten Primaria. [Internet] 2019 [Citado 2019 Nov 06];51(1):40-46. Disponible en: https://doi.org/10.1016/j.aprim.2018.05.004

10. MacDonald NESAGE Working Group on Vaccine Hesitancy. Vaccine hesitancy: Definition, scope and determinants. Vaccine. [Internet] 2015 [Citado 2019 Nov 06] 2015;33(34):4161-4. Disponible en:

https://doi.org/10.1016/j.vaccine.2015.04.036

11. Diccionario Inglés/Español. Barcelona: Editorial Océano. p 199.

12. Gagneur A, et al. Promoting vaccination in maternity wards motivational interview technique reduces hesitancy and enhances intention to vaccinate, results from a multicentre non-controlled pre- and post-intervention RCT- 
nested study, Quebec, March 2014 to February 2015. Euro Surveill. [Internet] 2019. [Citado 2019 Oct 11];24(36). Disponible en: https://doi.org/10.2807/1560-7917.ES.2019.24.36.1800641.

13. Luyten J, Bruyneel L, van Hoek AJ. Assessing vaccine hesitancy in the UK population using a generalized vaccine hesitancy survey instrument. Vaccine. [Internet] 2019. [Citado 2019 Oct 11];37(18):2494-2501. Disponible en: https://doi.org/10.1016/j.vaccine.2019.03.041.

14. Larson HJ, Jarrett C, Schulz WS, Chaudhuri M, Zhou Y, Dube E, Schuster M, MacDonald NE, Wilson R. Measuring vaccine hesitancy: The development of a survey tool. Vaccine. [Internet] 2015. [Citado 2019 Oct 14]:33(34):4165-4175. Disponible en: https://doi.org/10.1016/j.vaccine.2015.04.037.

15. Dubé $\grave{E}$, et al. Overview of knowledge, attitudes, beliefs, vaccine hesitancy and vaccine acceptance among mothers of infants in Quebec, Canada. Hum Vaccin Immunother. [Internet] 2019. [Citado 2019 Oct 14];15(1):113-120. Disponible en: https://doi.org/10.1080/21645515.2018.1509647

16. Cunningham RM, et al. Development of a Spanish version of the parent attitudes about childhood vaccines survey. Hum Vaccin Immunother. [Internet] 2019. [Citado 2019 Oct 15];15(5):1106-1010. Disponible en: https://doi.org/10.1080/21645515.2019.1578599

17. Yu Hu, Yaping Chen, Hui Liang, Ying Wang. Reliability and validity of a survey to identify vaccine hesitancy among parents in Changxing county, Zhejiang province. Hum Vaccin Immunother. [Internet] 2019. [Citado 2019 Oct 15];15(5):1092-1099. Disponible en http://doi.org/10.1080/21645515.2019.1572409

18. Roberts JR, et al. Vaccine hesitancy among parents of adolescents and its association with vaccine uptake. Vaccine. [Internet] 2015. [Citado 2019 Oct 16];33(14):1748-1755. Disponible en:

https://doi.org/10.1016/j.vaccine.2015.01.068

19. Henrickson NB, et al. Physician Communication Training and Parental Vaccine Hesitancy: A Randomized Trial. Pediatrics. [Internet] 2015. [Citado 2019 Oct 17];136(1):70-19. Disponible en: https://doi.org/10.1542/peds.2014-3199

20. Henrikson NB, Anderson ML, Opel DJ, Dunn J, Marcuse EK, Grossman DC. Longitudinal Trends in Vaccine Hesitancy in a Cohort of Mothers Surveyed in Washington State, 2013-2015. Public Health Reports. [Internet] 2017. [Citado 2019 Oct 21];132(4):451-454. Disponible en: https://doi.org/10.1177/0033354917711175

21. Opel DJ, Henrikson N, Lepere K, et al. Previsit Screening for Parental Vaccine Hesitancy: A Cluster Randomized Trial. Pediatrics. [Internet] 2019 [Citado 2019 Oct 21];144(5). Disponible en: https://doi.org/10.1542/peds.2019-0802

22. Abd Halim H, Abdul-Razak S, Md Yasin M, Isa MR. Validation study of the Parent Attitudes About Childhood Vaccines (PACV) questionnaire: The Malay version. Hum Vaccin Immunother. [Internet] 2019 [Citado 2019 Oct 22]. Disponible en: https://doi.org/10.1080/21645515.2019.1674112

23. Oladejo O, Allen K, Amin A, Frew PM, Bednarczyk RA, Omer SB. Comparative analysis of the Parent Attitudes about Childhood Vaccines (PACV) short scale and the five categories of vaccine acceptance identified by Gust et al. [Internet] Vaccine. 2016. [Citado 2019 Oct 24];34(41):4964-4968. Disponible en:

https://doi.org/10.1016/j.vaccine.2016.08.046

24. Domek GJ. Measuring vaccine hesitancy: Field testing the WHO SAGE Working Group on Vaccine Hesitancy survey tool in Guatemala. Vaccine. [Internet] 2018. [Citado 2019 Oct 25];36(35):5273-5281. Disponible en: https://doi.org/10.1016/j.vaccine.2018.07.046 
25. Mohd Azizi FS, Kew Y, Moy FM. Vaccine hesitancy among parents in a multi-ethnic country, Malaysia. Vaccine [Internet]. 2017 May 19 [Citado 2019 Oct 25];35(22):2955-2961. Disponible en: https://doi.org/10.1016/j.vaccine.2017.04.010.

26. Connors JT, Hodges EA, D'auria J, Windham L. Implementing vaccine hesitancy screening for targeted education. J Am Acad Nurse Pract. 2018. [Internet]. 2018 [Citado 2019 Oct 28];30(8):450-459. Disponible en: https://doi.org/10.1097/JXX.0000000000000056

27. Hofstetter AM, et al. Parental Vaccine Hesitancy and Declination of Influenza Vaccination Among Hospitalized Children. HOSPITAL PEDIATRICS. [Internet] 2018. [Citado 2019 Oct 29];8(10):6628-6635. Disponible en: https://doi.org/10.1542/hpeds.2018-0025

28. Rey D, Fressard L, Cortaredona S, Bocquier A, Gautier A, Peretti-Watel P, Verger P, on behalf of the Baromètre santé 2016 group. Vaccine hesitancy in the French population in 2016, and its association with vaccine uptake and perceived vaccine risk-benefit balance. Euro Surveill. [Internet] 2018 [Citado 2019 Oct 30];23(17):pii=17-00816. Disponible en: https://doi.org/10.2807/1560-7917.ES.23.17.17-00816

29. Napolitano F, D'Alessandro A, Angelillo IF. Investigating Italian parents' vaccine hesitancy: A cross-sectional survey. Hum Vaccin Immunother. [Internet] 2018. [Citado 2019 Nov 04];14(7):1558-1565. Disponible en: https://doi.org/10.1080/21645515.2018.146394

30. Cunningham, Rachel M. et al. Prevalence of Vaccine Hesitancy Among Expectant Mothers in Houston, Texas. Academic Pediatrics. [Internet] 2018 [Citado 2019 Nov 04];18(2):154-160. Disponible en: https://doi.org/10.1016/j.acap.2017.08.003

Cómo citar este artículo: Bernal-Vaquera BM, Morales-Jinez A, Moreno-Pérez NE. Indecisión a las vacunas: una revisión sistemática para abordar el fenómeno en Latinoamérica. SANUS [Internet]. 2021 [citado el $\underline{\mathrm{dd}} \underline{\mathrm{mm}} \underline{\text { aa] } ; ~ 6: ~}$ e182. Disponible en: https://doi.org/10.36789/sanus.vi1.182 